\title{
Test pilote de solutions pour des soins maternels respectueux: conclusions de recherches d'une etude menee en Zambie
}

Breakthrough RESEARCH

Follow this and additional works at: https://knowledgecommons.popcouncil.org/departments_sbsr-rh How does access to this work benefit you? Let us know!

\section{Recommended Citation}

Breakthrough RESEARCH. 2020. «Test pilote de solutions pour des soins maternels respectueux: conclusions de recherches d'une étude menée en Zambie, » Dossier de recherche programmatique. Washington, DC: Population Council. 


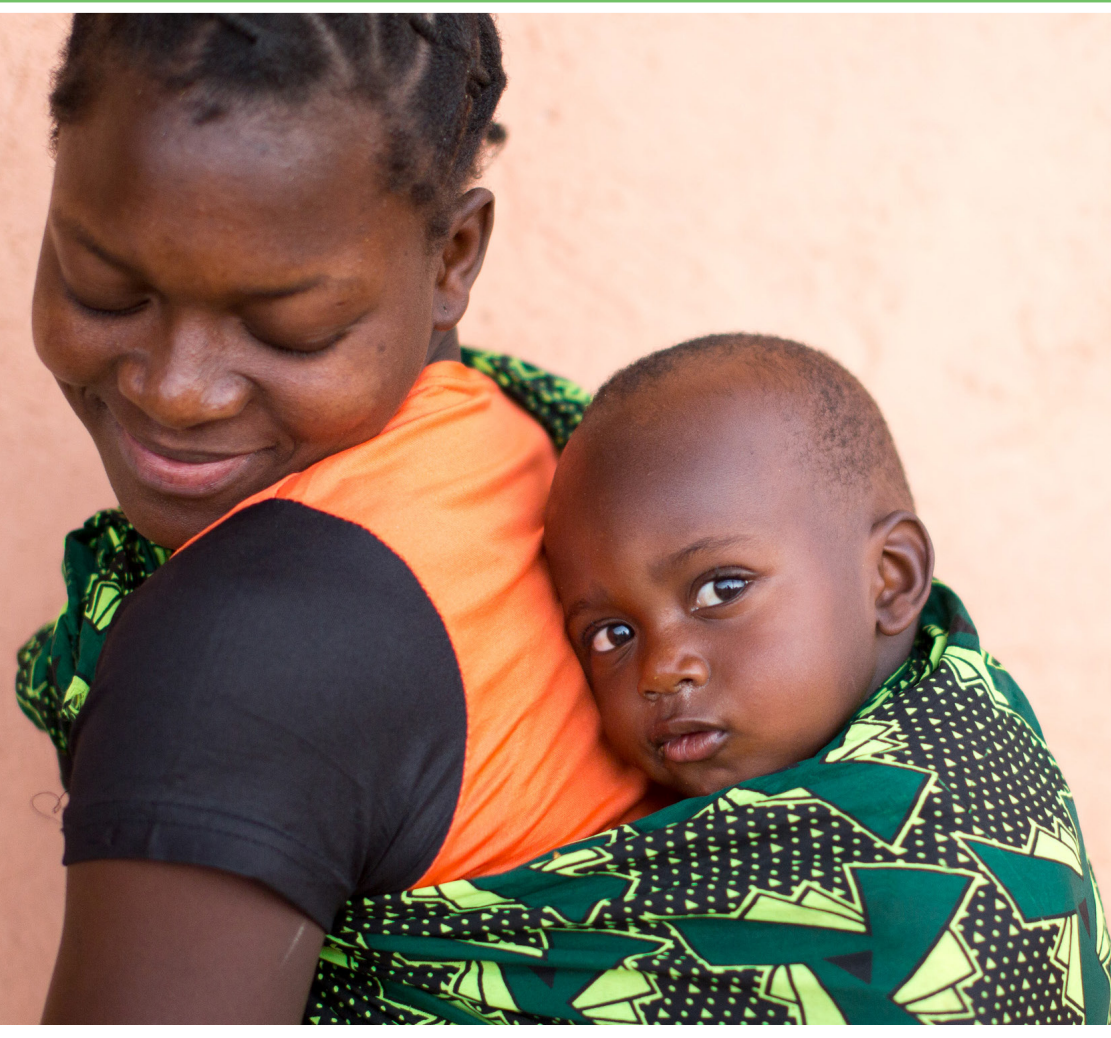

\section{TEST PILOTE DE SOLUTIONS POUR DES SOINS} MATERNELS RESPECTUEUX : CONCLUSIONS DE RECHERCHES D'UNE ÉTUDE MENÉE EN ZAMBIE

Cette synthèse de recherche détaille la mise en œuvre d'une phase pilote de solutions pour des soins maternels respectueux (SMR) à Chipata, en Zambie dans le cadre du projet Breakthrough RESEARCH. Elle décrit le modèle de la phase pilote, le processus de mise en œuvre par le partenaire local Safe Motherhood 360+ (SM360+) et les principaux enseignements tirés de la phase d'exécution. Elle fait suite à une précédente note de synthèse qui décrivait les conclusions de la recherche formative et les solutions développées pour promouvoir les SMR à l'aide d'un modèle comportemental dans la même localité.

\section{POINTS CLÉS}

Ces solutions, conçues par le biais d'un processus itératif fondé sur un modèle comportemental, étaient simples à mettre en ceuvre par les prestataires. En outre, les outils étaient facilement intégrés dans les services existants par les agents de santé durant la phase de travail et l'accouchement.

Le rôle joué par Safe Motherhood 360+ dans l'engagement des parties prenantes locales et les efforts de plaidoyer pour les SMR a été déterminant pour obtenir l'adhésion des plus hautes instances du gouvernement.

Cet ensemble de solutions, et plus particulièrement la boîte à outils BETTER, pour la prise en charge de la douleur, témoigne d'un certain potentiel dès qu'il s'agit d'augmenter la fréquence des encouragements et des interactions non-cliniques entre le prestataire et la cliente. 


\section{Aperçu}

Les SMR, tels que définis par l'Organisation mondiale de la Santé, renvoient aux soins « organisés et fournis à toutes les femmes d'une manière qui préserve leur dignité, leur vie privée et leur confidentialité, garantit l'absence de préjudice et de mauvais traitements et les aide à prendre des décisions éclairées et à bénéficier d'un soutien continu durant la phase de travail et l'accouchement (soulignement ajouté). »1

Outre les conséquences négatives sur le plan clinique, des soins maternels irrespectueux ont des conséquences néfastes sur l'expérience des femmes au moment de l'accouchement et leur comportement ultérieur en matière de recherche de soins, cette situation pouvant dissuader certaines femmes d'accoucher à l'avenir dans une structure de santé.

Toutefois, une étude réalisée en 2019 dans quatre pays a montré que $35 \%$ des femmes interrogées avaient été victimes « d'abus physique ou verbal, ou de stigmatisation ou de discrimination » durant le travail ou l'accouchement. ${ }^{2}$ Outre les conséquences négatives sur le plan clinique, des soins maternels irrespectueux ont des conséquences néfastes sur l'expérience des femmes au moment de l'accouchement et leur comportement ultérieur en matière de recherche de soins, cela pouvant dissuader certaines femmes d'accoucher à l'avenir dans une structure de santé. . $^{3,4}$

En 2018, ideas42, un partenaire de Breakthrough RESEARCH, a procédé à une recherche qualitative en étroite collaboration avec le projet SM360+ et avec le soutien de I'USAID/ Zambie pour déterminer les obstacles comportementaux empêchant les prestataires de dispenser systématiquement des SMR. En s'appuyant sur les résultats de cette recherche, ideas42 a co-créé un ensemble de solutions visant à promouvoir les SMR avec I'Office sanitaire du district de Chipata (OSD), les prestataires, les superviseurs et les femmes en phase de travail par le biais d'une phase itérative de test auprès des utilisateurs.

L'ensemble des solutions retenues étaient la boîte à outils BETTER,) pour la prise en charge de la douleur, une promesse prestataire-cliente et une boîte à retours (décrite dans l'encadré 1). Un résumé des conclusions de cette recherche allant des tests auprès des utilisateurs aux descriptions de ces solutions figure dans un précédent dossier. ${ }^{5}$ Pour faciliter la mise en œuvre et accroître l'efficacité de ces solutions, l'équipe a proposé de mettre en œuvre deux options supplémentaires : le fonds Fresh Start pour l'amélioration des structures de santé et un atelier de réflexion portant sur les valeurs et certaines clarifications.

Étant donné les résultats positifs des tests auprès des utilisateurs et des retours encourageant de la part des parties prenantes, notamment le Ministère de la Santé, I'OSD, I'USAID/Zambie et SM360+, il a été prévu de procéder à une phase pilote à petite échelle en 2019.

Ce dossier décrit le modèle de la phase pilote, le processus de mise en œuvre des solutions, les principaux enseignements tirés de la phase initiale et les prochaines étapes. Une prochaine note de synthèse détaillera les résultats de

\section{ENCADRÉ 1 SOLUTIONS DE SMR}

Vous trouverez ci-dessous une liste complète des solutions comprises dans l'intervention de SMR. Pour en savoir plus sur les solutions et les obstacles comportementaux auxquels elles tentent de remédier, veuillez consulter notre dossier précédent.

Boîte à outils BETTER pour la prise en charge de la douleur : BETTER est un acronyme anglais signifiant « Meilleur», formé à partir des mots « Breathe » (respirer), « Encourage » (encourager), « Turn » (se retourner), «Think» (penser) et «Rub» (masser). Cette boîte à outils a pour but d'incorporer la gestion de la douleur dans les soins de routine dispensés aux clientes. Elle comprend : (1) les affiches BETTER sur la technique de soulagement de la douleur, (2) le manuel BETTER sur la prise en charge de la douleur, (3) des balles de massage et (4) le guide BETTER du partogramme pour le soulagement de la douleur.

Boîte de retours : à leur sortie de la maternité, les femmes recevaient un jeton qu'elles devaient insérer dans l'une des fentes de la boîte reflétant le mieux le service dispensé.

Promesse prestataire-cliente : Une promesse faite entre le prestataire et la patiente au moment de l'admission dans la salle de travail et d'accouchement.

Fonds Fresh Start : Les structures de santé ont reçu un financement restreint qu'elles ont utilisé pour procéder à de petits changements dans la salle de travail afin d'améliorer l'expérience des patientes.

Atelier de réflexion : Un atelier visant à formuler l'intention de changer la manière dont les soins sont dispensés dans la structure et à introduire diverses solutions. 
I'évaluation et offrira un aperçu de l'efficacité des solutions visant à améliorer l'expérience des femmes quant aux soins qu'elles reçoivent durant l'accouchement.

\section{Préparation à une mise en œuvre réussie}

\section{Un plaidoyer pour des soins maternels respectueux}

Pour préparer la mise en œuvre et positionner ce travail en vue d'intensifier son impact à plus grande échelle, notre partenaire SM360+ a impliqué un large éventail de parties prenantes clés concernant l'importance des SMR. Par exemple, SM360+ a partagé ce travail avec les groupes de travail technique de l'initiative pour une maternité sans risque et I'assurance qualité pour plaider en faveur des SMR et en faire un sujet prioritaire.

Outre son travail de proximité, l'Association des sages-femmes de Zambie, qui a bénéficié d'un financement externe pour développer des directives nationales, a invité SM360+ à contribuer aux directives. SM360+ a présenté les conclusions des travaux de recherche et l'ensemble de solutions à ce groupe avant d'examiner la version préliminaire des directives nationales. Par conséquent, la version préliminaire des directives inclut désormais des approches non-cliniques (comme le fait d'encourager les cliniciens à utiliser des méthodes non-pharmacologiques pour soulager les douleurs, notamment des massages du dos, des techniques de respiration et certaines formes de distraction) en matière de SMR qui prennent en compte l'environnement dans lequel les prestataires évoluent.

Le Ministère de la Santé a également été impliqué dans le programme pour le développement et la mise en œuvre des solutions, tout comme la Direction nationale de l'assurance qualité, qui s'est intéressée à l'approche SMR. Pour garantir l'adhésion de l'OSD, ces entités ont été impliquées à travers toutes les phases du projet, allant du diagnostic du problème à la conception de l'ensemble de solutions. L'OSD compris à quel point il était important de promouvoir les SMR et reconnu que les solutions envisagées ne perturberaient pas la prestation de services de routine. Ainsi, la promesse prestataire-cliente pourrait être intégrée dans le processus d'admission actuel des clientes. L'appui de l'OSD a également permis au projet d'accéder aux structures de santé et aux encadreurs cliniques de SM360+ pour qu'ils prennent part à la mise en œuvre de l'ensemble de solutions. L'OSD a maintenu son engagement tout au long de la mise en œuvre, alors que SM360+ participait aux réunions de gestion mensuelles de l'OSD à Chipata.

\section{ENCADRÉ 2 MODÈLE PILOTE}

Une phase pilote de l'intervention a eu lieu pour tirer certains enseignements de la mise en œuvre des solutions et mieux comprendre leur efficacité entre les mois d'octobre et de décembre 2019.

Cinq établissements ont été sélectionnés pour la phase de mise en œuvre, notamment des structures de santé en milieu urbain et rural. Cinq établissements sanitaires supplémentaires ont été sélectionnés comme structures correspondantes à des fins de comparaison et n'ont pas bénéficié de l'intervention dans le cadre du modèle d'évaluation.

La méthodologie d'évaluation a été développée de manière collaborative avec SM360+ pour veiller à ce que les résultats de la phase pilote puissent être utilisés pour améliorer les programmes nationaux ultérieurs et appuyer les efforts visant à promouvoir les SMR à plus grande échelle.

\section{Mise en service des solutions}

Une formation des formateurs a été utilisée pour, dans un premier temps, présenter les solutions aux encadreurs cliniques de SM360+ qui ont ensuite organisé des ateliers de réflexion similaires pour former les prestataires. Le processus de mise en œuvre est décrit à la page suivante. L'atelier de réflexion destiné aux prestataires avait pour but de susciter un changement, selon le concept de la théorie du changement de l'ensemble de solutions, à l'aide des moyens suivants : (i) générer des réflexions sur l'état actuel des soins en donnant un espace aux prestataires pour faire part des défis rencontrés au moment de dispenser des SMR, (ii) les motiver à changer l'état des soins en procédant à des jeux visant à accroître leur degré d'empathie et à discuter de l'impact des soins sur l'expérience vécue par les clientes, et (iii) consolider l'engagement en faveur du changement en fixant des objectifs individuels et conjoints au sein des structures de santé. Les ateliers ont été conçus pour être interactifs et comprenaient des scénarios de jeux de rôle dans le contexte d'un accouchement et des jeux participatifs mettant en application les diverses solutions.

Durant l'atelier de réflexion, les prestataires étaient également informés sur les fonds Fresh Start, une composante de l'ensemble de solutions soutenue par l'intermédiaire du projet. Chaque structure recevait un financement restreint à

aL'approche de formation des formateurs renvoie à la stratégie consistant à utiliser des formateurs de niveau supérieur chargés d'instruire de nouveaux formateurs sur un sujet ou une compétence donné. Les nouveaux formateurs sont ensuite chargés d'organiser les ateliers de formation ultérieurs sur les divers supports. 
la suite de l'atelier de réflexion pour procéder à des changements au sein de l'établissement et améliorer l'expérience des clientes d'une quelconque manière décidée collectivement par la structure. Les superviseurs et les prestataires des structures devaient s'aligner sur la meilleure utilisation des fonds. Cette solution avait pour but de responsabiliser chaque établissement et ses prestataires pour en faire des agents du changement dans le processus de transformation de l'état actuel des soins. De plus, étant donné que toutes les structures différaient en termes de gestion et de contexte, cette solution a permis aux établissements sanitaires de prendre des décisions sur les aspects à prioriser plutôt que d'imposer des solutions générales de manière descendante. Les détails concernant la manière dont ces fonds ont été utilisés et les enseignements tirés de cette approche figurent plus loin dans ce dossier.

\section{Processus de mise en œuvre}

\section{Atelier de réflexion initial}

L'atelier a été animé par ideas42 à Chipata en Zambie, en collaboration avec le personnel de SM360+, notamment les accompagnateurs cliniques. Des représentants des établissements ont été invités à participer avec des délégués provinciaux de l'Association des sages-femmes de Zambie et du bureau sanitaire provincial du chef du personnel infirmier. L'atelier s'est déroulé sur deux jours.

\section{Ateliers de réplication et de réflexion}

Les encadreurs cliniques ont participé aux ateliers de réflexion et organisé des ateliers de réplication d'une journée destinés aux cliniciens et au personnel intervenant durant la phase de travail et l'accouchement dans chacune des cinq structures de santé retenues pour la mise en œuvre. Les encadreurs cliniques ont reçu des supports de formation et des guides de facilitation, ainsi que l'ensemble finalisé de solutions, notamment la boîte à outils de gestion de la douleur BETTER, la boîte à retours et les affiches. Si un prestataire n'était pas présent à l'atelier de réplication, l'encadreur clinique utilisait un guide complémentaire pour orienter cette personne sur ces solutions à une date ultérieure.

\section{Introduction du fonds Fresh Start}

Peu de temps après les ateliers de réplication, et avec le soutien des encadreurs cliniques, les structures sanitaires ont organisé une réunion avec les superviseurs et les prestataires pour examiner la prestation de services et l'état des établissements, et déterminer l'usage adéquat des fonds. Les structures ont ensuite été informées d'utiliser les fonds dans les deux semaines à venir.

\section{Suivi continu}

Les encadreurs cliniques de SM360+ ont dispensé un soutien continu aux structures de santé, tandis que les superviseurs de l'OSD assuraient l'encadrement et les visites de supervision formative. Les encadreurs cliniques ont inclus la supervision et le suivi des modèles dans leurs cahiers des charges existants et dans le cadre de leurs visites. Par ailleurs, les agents de collecte de données ont réalisé des visites d'observation pour comprendre comment les modèles étaient utilisés dans la pratique.

\section{Évaluation de la phase pilote}

Pour tirer certains enseignements de la phase de mise en œuvre et de l'efficacité potentielle des solutions, une évaluation de la phase pilote a été réalisée.

\section{Principaux enseignements}

Vous trouverez ci-dessous plusieurs points à retenir concernant la mise en œuvre de l'ensemble de solutions. Ceux-ci ont été identifiés au fil de conversations avec le personnel de SM360+ chargé de cette étape et les thèmes préliminaires des entretiens avec le personnel des établissements. Dans la mesure où les solutions n'ont pas été appliquées dans nos établissements correspondants, les enseignements répertoriés ci-après reflètent uniquement l'expérience vécue au sein des cinq sites de mise en œuvre. La prochaine note de synthèse décrira les résultats complets de l'évaluation une fois l'analyse des données terminée.

Les solutions ont incité les superviseurs

à procéder à d'autres changements pour améliorer les soins administrés aux

patientes, dépassant le cadre des solutions à proprement parler.

\section{Réactions générales de l'établissement et de I'OSD}

Les structures de santé mettent en œuvre l'ensemble de solutions depuis octobre 2019. Les responsables des structures de santé ou les superviseurs des maternités ont apprécié le fait que les modèles étaient simples et faciles à mettre en œuvre par les prestataires. Les responsables ont également mis en avant la manière dont chacune des solutions agissait en complément d'une autre, cela permettant d'améliorer l'administration des soins par les prestataires. Par exemple, I'utilisation de la promesse prestataire-cliente 
a facilité l'usage par les agents des outils de gestion des douleurs BETTER. Et un responsable de déclarer :

[Les modèles] ne fonctionnent pas
en simultané; ils opèrent à différents
moments mais ils sont tous utiles... Si je
dis que je retire la boîte à retours, comment
allons-nous savoir si les clientes sont satis-
faites ou non ? Peut-être que les promesses
faites aux clientes par le prestataire n'ont pas
été honorées et cela peut se refléter dans
les retours laissés (...) Sans boîte à retours,
comment saurons-nous si les clientes sont
satisfaites ou non avec nos services ? Et s'il
n'y a pas de promesse prestataire-cliente,
comment la cliente saura-t-elle qu'elle est
censée être traitée de manière respectueuse?

Un résultat positif non-anticipé réside dans le fait que les modèles ont amené les structures à envisager d'autres moyens d'améliorer l'expérience des femmes en matière de soins. Par exemple, le responsable d'un établissement a été inspiré par les techniques BETTER de gestion des douleurs et a suggéré d'accrocher des affiches dans la salle de travail illustrant une famille heureuse pour mieux distraire les femmes durant des accouchements douloureux. Un autre responsable a suggéré d'éduquer les femmes enceintes durant les consultations prénatales sur ce qu'elles doivent anticiper durant la phase de travail et l'accouchement en leur faisant visiter la salle de travail.

L'OSD a également fait part de son enthousiasme concernant l'impact que les modèles pourraient exercer sur l'amélioration de la qualité des soins etse montre particulièrement intéressé quant à la manière dont la meilleure expérience des clientes peut influencer leur comportement en matière de recherche de soins. Les responsables ont trouvé la promesse

\section{TABLEAU 1 UTILISATION DES FONDS FRESH START PAR STRUCTURE}

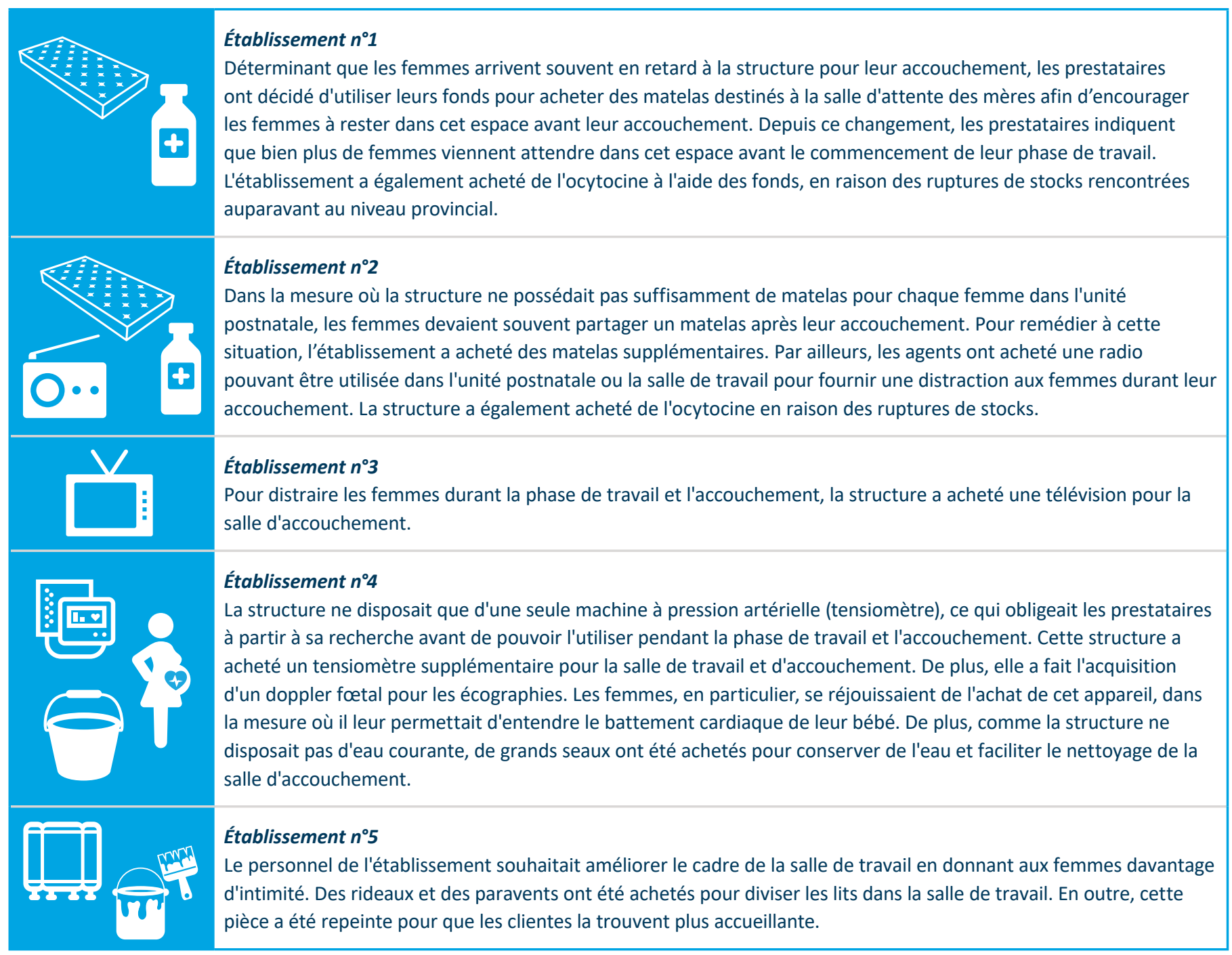


prestataire-cliente et son impact sur ces deux domaines particulièrement encourageant et ont commencé à réfléchir à la manière dont elle pouvait être adoptée à plus grande échelle. Ils se réjouissaient également de la boîte à retours, dans la mesure où elle donnait aux clientes la possibilité d'exprimer leur satisfaction de manière anonyme.

\section{Résultats des ateliers de réflexion et de réplication}

La formation des formateurs a constitué une approche efficace pour former les prestataires de soins dans la mesure où elle créait un environnement sûr. Les prestataires se sentaient à l'aise pour poser des questions et avoir des discussions honnêtes au sujet des interactions avec les clientes, étant donné l'approche par les pairs de la formation. Le personnel de SM360+ en charge de la mise en œuvre a relevé que cette approche par les pairs était particulièrement efficace par rapport à d'autres modes de formation descendants. Les prestataires ont également indiqué qu'ils aimaient le format interactif des ateliers et mentionné que cet aspect leur avait permis de réfléchir aux soins qu'ils dispensaient. Les encadreurs cliniques ont apprécié le fait que les ateliers se tenaient du côté des structures plutôt que de faire déplacer le personnel, permettant ainsi aux prestataires de se concentrer et de s'engager.

Plusieurs responsables ont rapporté que les ateliers de réplication avaient changé la manière dont les prestataires comprenaient leur rôle pendant la phase de travail et l'accouchement. Les prestataires comprenaient qu'ils devaient être conscients des instructions données aux clientes, dans la mesure où cela les amenait à coopérer davantage. Un autre responsable a noté que de nombreux prestataires connaissaient déjà certaines techniques de gestion des douleurs mais ne les avaient pas mis en œuvre. L'atelier a renforcé leur importance.

\section{Utilisation des fonds Fresh Start}

Dans toutes les structures, les responsables ont demandé aux prestataires de réfléchir à la manière dont ils allaient prioriser I'utilisation des fonds Fresh Start. Par la suite, une réunion a été organisée avec l'équipe des finances de chaque établissement pour voter et décider de la meilleure utilisation des fonds. Le tableau 1 décrit la manière dont les fonds ont été utilisés au sein de chaque structure participant à la mise en œuvre.

L'utilisation des fonds Fresh Start démontre comment chaque structure envisageait d'améliorer l'expérience des patientes dans leur cadre spécifique. Ces contributions ont également montré à quel point il était difficile de mettre l'accent sur une meilleure expérience des clientes quand ils existent d'autres
Un responsable a remarqué qu'une cliente lui

avait confié « ne même pas avoir le sentiment

d'accoucher » grâce au soutien reçu pour

soulager les douleurs.

exigences sur le plan clinique. Par exemple, les récentes ruptures de stocks ont amené les structures à utiliser des fonds pour acheter de l'ocytocine qui, bien que d'une importance critique dans la prévention et la prise en charge des complications pendant la phase de travail, ne contribue pas à l'amélioration de l'expérience non-clinique des clientes.

\section{Réponse à la promesse prestataire-cliente}

Certains prestataires n'étaient initialement pas convaincus par la promesse prestataire-cliente et partaient du principe que les patientes refuseraient de signer le document. Les prestataires ont été encouragés par les encadreurs cliniques à démarrer la mise en œuvre et à ne pas envisager un refus des clientes, étant donné que durant les tests auprès des utilisateurs, cette question n'avait pas été soulevée. Au lancement de la phase de mise en œuvre, les prestataires se sont réjouis de cette promesse et ont fait part de leur satisfaction, en recommandant la poursuite de cette pratique après la phase pilote. Après avoir signé l'accord, les prestataires ont affirmé qu'ils se sentaient liés par ces engagements et qu'ils leur donneraient matière à réflexion lors de la prestation de soins.

Comme prévu, les femmes du Groupe d'action pour une maternité sans risque (SMAG) ont partagé la promesse prestataire-cliente avec certaines femmes avant leur arrivée dans la salle de travail et d'accouchement par le biais d'agents de santé communautaires et durant les consultations prénatales, ainsi qu'au moment de leur admission à la maternité. Toutefois, dans une des structures, cette pratique a suscité une certaine confusion : Les prestataires n'ont pas utilisé ce document au moment de l'admission des clientes à la maternité, pensant que cela n'était pas nécessaire dans la mesure où elles avaient déjà reçu la promesse durant leurs consultations prénatales. Dans d'autres établissements, ce défi n'a pas été rencontré et les prestataires ont honoré la promesse de manière systématique lors des accouchements.

\section{Points à retenir concernant la boîte à outils de gestion dela douleur BETTER}

Les prestataires ont indiqué que le recours à la boîte à outils BETTER pour la gestion de la douleur avait augmenté le nombre d'interactions avec les clientes. Avant d'utiliser cette boîte à outils, un responsable a déclaré que les prestataires ne 
passaient pas beaucoup de temps avec les clientes en dehors des tâches d'ordre clinique. Néanmoins, avec la boîte à outils pour la gestion de la douleur et le guide du partogramme BETTER, un responsable a indiqué que les prestataires venaient plus fréquemment en aide aux clientes. Un autre responsable a remarqué qu'une cliente lui avait confié « ne même pas avoir le sentiment d'accoucher » grâce au soutien reçu pour soulager les douleurs.

Les prestataires ont indiqué que les outils BETTER étaient très utiles et ont apprécié que certaines techniques puissent être utilisées par les clientes elles-mêmes ou avec le soutien d'un groupe SMAG, dans la mesure où les prestataires sont par moments très occupés. Bon nombre de prestataires aimaient les balles de massage, une composante de la boîte à outils à outil BETTER, et ont souligné le fait qu'il s'agissait de la technique préférée des clientes. Un prestataire a d'ailleurs souligné que la balle « faisait des merveilles » pour soulager les douleurs.

\section{Réponse à la boîte de retours}

La boîte à retours était une des solutions les plus difficiles à mettre en œuvre avec fidélité. Durant la mise en œuvre, lorsque la boîte à retours a été présentée, certains prestataires se sont montrés hésitants car ils s'attendaient principalement à des messages négatifs.

Toutefois, les prestataires ont indiqué que les clientes appréciaient davantage leurs services que ce qu'ils avaient envisagé à l'origine. Durant les observations des agents de collecte de données au sein des structures, les prestataires semblaient donner des instructions claires et sans parti pris quant à la manière d'utiliser les boîtes à retours et ce que chacune des trois fentes représentait. De plus, dans la plupart des structures, les retours ont été examinés chaque mois dans le cadre de réunions régulières. Certains défis ont été cités, notamment concernant la manière dont la boîte à retours était comprise par la communauté et sa gestion globale, montrant ainsi qu'il était possible de tirer meilleur parti des SMAG pour partager le but de cet outil avec la communauté.

\section{Étapes suivantes}

Les leçons tirées de la phase pilote suggèrent que les solutions affichent un certain potentiel en termes de promotion des SMR, tout en mettant en lumière plusieurs améliorations éventuelles en vue d'une intensification à plus grande échelle. Par exemple, les résultats de la phase pilote ont souligné le rôle que des SMAG et d'autres acteurs communautaires peuvent jouer pour faciliter la compréhension des solutions dans le contexte communautaire.
La stratégie consistant à donner une certaine latitude aux structures pour procéder à des améliorations durant la phase pilote et partager ces adaptations s'est avérée payante et a garanti le développement continu de solutions avec les prestataires et les superviseurs menant directement leur mise en œuvre. L'engagement continu de SM360+ auprès de I'OSD a également été déterminant dans la réussite de la phase pilote.

Dans le cadre de cette phase, les données de base et finales ont été recueillies auprès des prestataires et des clientes pour évaluer l'efficacité des solutions. Ces résultats et certaines leçons complémentaires seront répertoriés dans un dossier ultérieur. Les leçons tirées de la mise en œuvre décrites dans ce dossier, ainsi que les observations quasi-expérimentales de l'évaluation qui seront intégrés au prochain dossier, contribueront à affiner les solutions pour accroître leur faisabilité et leur impact. Une fois l'évaluation réalisée, les résultats seront partagés et débattus avec l'OSD et le Ministère de la Santé avec le soutien de SM360+. 


\section{RÉFÉRENCES}

${ }^{1}$ Bibliothèque de santé génésique de l'Organisation mondiale de la Santé (OMS). 2018. «WHO recommendation on respectful maternity care during labour and childbirth. » Genève: OMS. Disponible sur : https://extranet.who.int/rhl/topics/preconception-pregnancy-childbirth-and-postpartum-care/ care-during-childbirth/who-recommendation-respectful-maternity-care-during-labour-and-childbirth

${ }^{2}$ Bohren, M. A. et al. 2019. " How women are treated during facility-based childbirth in four countries: a cross-sectional study with labour observations and community-based surveys, » The Lancet 394(10210): 1750-1763. doi: 10.1016/S0140-6736(19)31992-0

${ }^{3}$ Kujawski, S. et al. 2015. " Association between disrespect and abuse during childbirth and women's confidence in health facilities in Tanzania, » Maternal and Child Health Journal 19(10): 2243-2250. doi: 10.1007/ s10995-015-1743-9

${ }^{4}$ Faye, A., M. Niane, \& I. Ba. 2011. " Home birth in women who have given birth at least once in a health facility: contributory factors in a developing country. » Acta Obstetricia et Gynecologica Scandinavica 90(11): 1239-43. doi: 10.1111/j.1600-0412.2011.01163.x

${ }^{5}$ Breakthrough RESEARCH. 2019. "Vers des soins maternels plus respectueux durant la phase de travail et l'accouchement ", Dossier de recherche programmatique. Washington, DC : Population Council. https:// breakthroughactionandresearch.org/wp-content/ uploads/2020/10/Respectful-Care-Brief.pdf

\section{Remerciements}

Ce dossier de recherche programmatique décrit le travail dirigé par ideas42 dans le cadre du projet Breakthrough RESEARCH. Ce dossier et le travail qu'il décrit sont rendus possibles grâce aux efforts et au soutien de SafeMotherhood360+, Population Council Zambia, et Population Council, Washington, DC.

\section{Citation suggérée :}

Breakthrough RESEARCH. 2020. «Test pilote de solutions pour des soins maternels respectueux : conclusions de recherches d'une étude menée en Zambie, » Dossier de recherche programmatique. Washington DC: Population Council.

Crédits photographiques de la page de couverture : (C)2015 Bread for the World (CC BY-ND 2.0/No modifications)

\section{Courriel}

BreakthroughResearch@popcouncil.org

\section{Breakthrough RESEARCH | Population Council}

4301 Connecticut Ave., NW, Suite 280 | Washington, DC 20008 +12022379400 | breakthroughactionandresearch.org

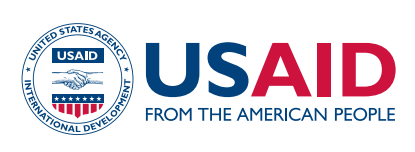

Breakthrough RESEARCH existe grâce au soutien généreux du peuple américain par l'intermédiaire de l'Agence des États-Unis pour le développement international (USAID) aux termes de I'accord de coopération N AID-OAA-A-17-00018. Le contenu de ce document relève de la responsabilité exclusive de Breakthrough RESEARCH et du Population Council et ne reflète pas nécessairement l'opinion de I'USAID ou celle du gouvernement des États-Unis.
POPULATION COUNCIL

Ideas. Evidence. Impact.

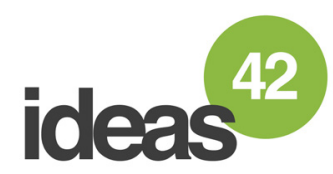

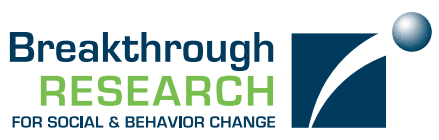

Notre projet transforme les données probantes en actions concrètes à l'aide d'un éclairage nouveau sur les questions relatives à l'amélioration des politiques et des programmes SMR, en cherchant à accroître le rapport coût-efficacité des stratégies de I'USAID en matière de santé et de développement. Breakthrough RESEARCH impulse des changements sociaux et comportementaux en réalisant des études et des évaluations inédites et en promouvant les solutions fondées sur des données probantes afin d'améliorer les programmes de santé et de développement à travers le monde. Breakthrough RESEARCH est un consortium dirigé par le Population Council en partenariat avec Avenir Health, ideas42, I'Institute for Reproductive Health de Georgetown University, le Population Reference Bureau et Tulane University. 